\title{
The Coronavirus Disease 2019 Epidemic Situation in China
}

\author{
Jin Wang, Zhihui Li and Jiahai Lu* \\ Department of Epidemiology, School of Public Health, Sun Yat-sen University, Guangzhou, Guangdong Province, 510080, China
}

It has been 17 years since the outbreak of severe acute respiratory syndrome (SARS) in 2003, which causes at least 8096 infections and 774 deaths worldwide. History repeats itself! In Dec 2019, China reported to the World Health Organization (WHO) a cluster of patients with pneumonia of unknown etiology in Wuhan, a major hub of transportation with a population of 11 million people. On Jan 9, 2020, a novel coronavirus (officially named as SARSCoV-2 on Feb 11) was identified as the cause of this outbreak. The number of infected cases increased sharply, and the epidemic rapidly spread throughout the country. The WHO on Jan 30 declared the outbreak a Public Health Emergency of International Concern. By Feb 15, 2020, the cumulative number of confirmed cases had reached 66,577, far exceeding the number of SARS cases in 2003. According to the released news, the case rate fatality is $2.3 \%(1,524 / 66,577)$. Geographically, the disease has spread beyond China to over 30 countries, with Japan having the most confirmed cases outside China.

The SARS-CoV-2 is mainly transmitted through respiratory droplets. After infection, patients showed lymphopenia and bilateral ground-glass opacity or consolidation in chest CT scans, along with common symptoms that include fever, dry cough, and shortness of breath, at the onset of illness. ${ }^{1,2}$ In severe cases, dyspnea, respiratory distress syndrome or septic shock may develop. ${ }^{2}$ Early observation of infections of health-care workers as well as family members has suggested that human-to-human transmission has occurred among close contacts. ${ }^{3,4}$ The epidemic doubled in size every 6.4-7.4 days in its early stage, with the basic reproductive number $\left(\mathrm{R}_{0}\right)$ estimated to be $2.2-2.68 .3,5,6$

Coronavirus is an enveloped, positive-strand RNA virus, belonging to Coronavirinae subfamily within Coronaviridae family. The coronaviruses can infect human, livestock, avian, bat, mouse and other wild animals. They can cause respiratory, gastrointestinal, hepatic and central nervous system diseases of varying severity, sometimes fatal. The two types of coronaviruses that we are currently familiar with are SARS-CoV in 2003 and Middle East respiratory syndrome coronavirus (MERS-CoV) broken out in 2012. The SARS-CoV-2 is the seventh known coronavirus that can infect humans. ${ }^{7}$ This virus seems to have greater infectivity (e.g., a higher R0) but a lower case fatality rate. ${ }^{8}$

Bats might be the original host of this virus. ${ }^{9}$ The SARS-CoV-2

Abbreviations: ACE-2, angiotensin-converting enzyme-2; CoV, coronavirus; $\mathrm{COV}$ ID-19, coronavirus disease 2019; CT, computed tomography; MERS-CoV, Middle East respiratory syndrome coronavirus; SARS, severe acute respiratory syndrome; WHO, World Health Organization.

Received: February 17, 2020; Revised: March 03, 2020; Accepted: March 04, 2020 *Correspondence to: Jiahai Lu, Department of Epidemiology, School of Public Health, Sun Yat-sen University, Guangzhou, Guangdong Province, 510080, China. Tel: +86-20 87332438,E-mail: lujiahai@mail.sysu.edu.cn

How to cite this article: Wang J, Li Z, Lu J. The Coronavirus Disease 2019 Epidemic Situation in China. Exploratory Research and Hypothesis in Medicine 2020;5(1):1-2. doi: 10.14218/ERHM.2020.00009. is a $\beta \mathrm{CoV}$ of group $2 \mathrm{~B}$, sharing $79.5 \%$ of genetic sequence with SARS-CoV and has $96.2 \%$ homology to a bat coronavirus. ${ }^{9,10}$ However, the possibility of direct transmission from bats to humans is small, and an intermediate host is often needed to mediate zoonoses from natural reservoir to terminal hosts. It is speculated that SARS-CoV-2 may come from a certain wild animal and spread rapidly in the Huanan Seafood Wholesale Market in Wuhan, where the viruses were originally isolated. Homology modelling analysis showed structural similarity between the receptor-binding domains of SARS-CoV and SARS-CoV-2 to the human angiotensinconverting enzyme-2 (ACE-2) receptor. ${ }^{10}$ SARS-CoV-2 has been considered to link to game consumption, a habit obsessive by some Chinese people. ${ }^{11}$ After the outbreak, the Chinese Government has closed the Huanan Seafood Wholesale Market in Wuhan and banned all forms of wild animal transaction.

Since the outbreak, geographical spread of the disease raises grave concerns around the world. The Chinese health authorities have taken unprecedented measures to control the source of infection. ${ }^{12}$ From Jan 23, Chinese authorities have imposed travel bans on Wuhan and several cities near Wuhan. Individuals are popularized the knowledge to take self-protection measures, such as good personal hygiene, fitted masks, ventilation and avoiding crowded places. There is likelihood that some mild or asymptomatic patients do not seek health care but as a source transmitting the virus to other humans, which may complicate or delay the effectiveness of infection-control measures. ${ }^{13}$ At present, there are no specific antivirals or vaccines although clinical trial of Lopinavir/ritonavir has been launched for SARS-CoV-2 (ChiCTR2000029308), and the management of infection is largely supportive. Thus, molecular diagnostic platforms for rapidly identifying SARS-CoV-2 as well as effective vaccine strategies are urgently needed to develop.

In this century, the coronaviruses attacked China twice, each time causing public panic, heavy deaths and huge economic losses. It seems that people have not yet established a rapid, effective response to public health threats. Although scientific research on the etiology and structural features of unknown pathogens is important, it is more practical to establish a strong and effective public health system that can assure the nation's health and safety. The investment in core public-health systems and infrastructure will no doubt be critical for preventing or controlling this kind of biosecurity. Local health departments need to strengthen their ability to mount an effective health emergency response, including identification of suspicious cases as well as individuals with high-risk exposures at the early stage, even in a sudden outbreak of unknown pathogens. Coordination and collaboration among surveillance departments, health sectors and laboratories are particularly important. In addition, both MERS and SARS nosocomial outbreaks are characterized by early nosocomial super-spreading events. Although so far no evidence indicates a super transmission event in the COVID-19 outbreak, the situation of nosocomial infection is still serious. As of Feb 11, 1,716 medical staffs had been infected 
in China, with 5 of them died. At present, it is still unclear how many patients were cross infected when they waited for diagnosis or treatment in fever clinic. Nosocomial infection may be a driving factor for the epidemic of infectious diseases. From this point of view, strict control of nosocomial infection is also significant in fighting the spread of epidemic diseases.

So far, the spread of the epidemic has begun to slow down. Many questions remain unknown, including animal reservoir, pathogenesis, epidemiology of SARS-CoV-2 as well as risks for globe transmission. There are some important things we need to think about, in particular, how to change from passive response to active surveillance before public health threats appear. At present, almost no surveillance system can effectively integrate human and animal disease information, which leads to the inability to detect new zoonotic diseases and evaluate the risk of spill-over from animals to humans. Thus, the establishment of an effective zoonotic disease surveillance system may be a crucial step helpful for spotting the emergence of a pandemic virus and warning the early spread of cross-species pathogens.

\section{Acknowledgments}

None.

Funding

This work was funded by the Key-Area Research and Development Program of Guangdong Province (2018B020241002), the National Science and Technology Major Project (No. 2018ZX10101002001-001) and the Guangdong Provincial Science and Technology Project (2020B111112003).

\section{Conflict of interest}

The authors declare that they have no conflict of interest.

\section{Author contributions}

JW and ZL wrote the first draft of the manuscript; JL summarized viewpoints of the article and corrected the manuscript. All authors contributed to the manuscript and approved the submitted version.

\section{References}

[1] Chen N, Zhou M, Dong X, Qu J, Gong F, Han Y, et al. Epidemiological and clinical characteristics of 99 cases of 2019 novel coronavirus pneumonia in Wuhan, China: a descriptive study. Lancet 2020;395(10223):507-513. doi:10.1016/S0140-6736(20)30211-7.

[2] Huang C, Wang Y, Li X, Ren L, Zhao J, Hu Y, et al. Clinical features of patients infected with 2019 novel coronavirus in Wuhan, China. Lancet 2020;395(10223):497-506. doi:10.1016/S0140-6736(20)30183-5.

[3] Li Q, Guan X, Wu P, Wang X, Zhou L, Tong Y, et al. Early Transmission Dynamics in Wuhan, China, of Novel Coronavirus-Infected Pneumonia. N Engl J Med 2020. doi:10.1056/NEJMoa2001316.

[4] Chan JF, Yuan S, Kok KH, To KK, Chu H, Yang J, et al. A familial cluster of pneumonia associated with the 2019 novel coronavirus indicating person-to-person transmission: a study of a family cluster. Lancet 2020;395(10223):514-523. doi:10.1016/S0140-6736(20)30154-9.

[5] Wu JT, Leung K, Leung GM. Nowcasting and forecasting the potential domestic and international spread of the 2019-nCoV outbreak originating in Wuhan, China: a modelling study. Lancet 2020;395(10225):689-697. doi:10.1016/S0140-6736(20)30260-9.

[6] Riou J, Althaus CL. Pattern of early human-to-human transmission of Wuhan 2019 novel coronavirus (2019-nCoV), December 2019 to January 2020. Euro Surveill 2020;25(4). doi:10.2807/1560-7917. ES.2020.25.4.2000058.

[7] Zhu N, Zhang D, Wang W, Li X, Yang B, Song J, et al. A Novel Coronavirus from Patients with Pneumonia in China, 2019. N Engl J Med 2020;382:727-733. doi:10.1056/NEJMoa2001017.

[8] del Rio C, Malani PN. 2019 Novel Coronavirus-Important Information for Clinicians. JAMA 2020. doi:10.1001/jama.2020.1490.

[9] Zhou P, Yang XL, Wang XG, Hu B, Zhang L, Zhang W, et al. A pneumonia outbreak associated with a new coronavirus of probable bat origin. Nature 2020;579:270-273. doi:10.1038/s41586-020-2012-7.

[10] Lu R, Zhao X, Li J, Niu P, Yang B, Wu H, et al. Genomic characterisation and epidemiology of 2019 novel coronavirus: implications for virus origins and receptor binding. Lancet 2020;395(10224):565-574. doi:10.1016/S0140-6736(20)30251-8.

[11] Li J, Li JJ, Xie X, Cai X, Huang J, Tian X, et al. Game consumption and the 2019 novel coronavirus. Lancet Infect Dis 2020;20(3):275-276. doi:10.1016/S1473-3099(20)30063-3.

[12] Nkengasong J. China's response to a novel coronavirus stands in stark contrast to the 2002 SARS outbreak response. Nat Med 2020;26:310311. doi:10.1038/s41591-020-0771-1.

[13] Munster VJ, Koopmans M, van Doremalen N, van Riel D, de Wit E. A Novel Coronavirus Emerging in China - Key Questions for Impact Assessment. N Engl J Med 2020;382:692-694. doi:10.1056/NEJMp 2000929. 